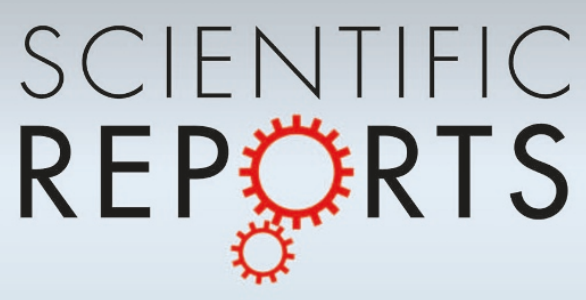

OPEN

SUBJECT AREAS:

MAGNETIC PROPERTIES

AND MATERIALS

SUPERCONDUCTORS

SUPERCONDUCTING PROPERTIES

AND MATERIALS

ELECTRONIC PROPERTIES AND

MATERIALS

Received

4 April 2013

Accepted

1 July 2013

Published

26 July 2013

Correspondence and requests for materials should be addressed to Y.K. (yoshiharu.k@lab. ntt.co.jp)

* On leave from Nagaoka University of Technology.

$\uparrow$ Current address: Department of Applied Physics, Tokyo University of Science,

1-3 Kagurazaka, Shinjuku, Tokyo 1628601 , Japan.

\section{Emerging superconductivity hidden beneath charge-transfer insulators}

\author{
Yoshiharu Krockenberger ${ }^{1}$, Hiroshi Irie', Osamu Matsumoto ${ }^{2}$, Keitaro Yamagami ${ }^{1 *}$, Masaya Mitsuhashi ${ }^{1 *}$, \\ Akio Tsukada'†, Michio Naito² \& Hideki Yamamoto' \\ ${ }^{1}$ NTT Basic Research Laboratories, NTT Corporation, 3-1 Morinosato-Wakamiya, Atsugi, Kanagawa 243-0198, Japan, \\ ${ }^{2}$ Department of Applied Physics, Tokyo University of Agriculture and Technology, 2-24-16 Naka-cho, Koganei, Tokyo 184-8588, \\ Japan.
}

In many of today's most interesting materials, strong interactions prevail upon the magnetic moments, the electrons, and the crystal lattice, forming strong links between these different aspects of the system.

Particularly, in two-dimensional cuprates, where copper is either five- or six-fold coordinated, superconductivity is commonly induced by chemical doping which is deemed to be mandatory by destruction of long-range antiferromagnetic order of $3 d^{9} \mathrm{Cu}^{2+}$ moments. Here we show that superconductivity can be induced in $\mathrm{Pr}_{2} \mathrm{CuO}_{4}$, where copper is four-fold coordinated. We induced this novel quantum state of $\mathrm{Pr}_{2} \mathrm{CuO}_{4}$ by realizing pristine square-planar coordinated copper in the copper-oxygen planes, thus, resulting in critical superconducting temperatures even higher than by chemical doping. Our results demonstrate new degrees of freedom, i.e., coordination of copper, for the manipulation of magnetic and superconducting order parameters in quantum materials.

W hile the issue of the mechanism of high temperature superconductivity continues to be controversial, one can clearly state that there have been many experimental results demonstrating that the lattices make a strong impact on the behavior of electrons ${ }^{1}$. First principle methods predict that square planar coordinated cuprates, e.g., $\mathrm{Pr}_{2} \mathrm{CuO}_{4}$ are expected to be metals ${ }^{2-4}$, unlike octahedral-coordinated cuprates. Square planar coordinated cuprates are those which, upon electron doping, become eventually high temperature superconductors subject to an annealing treatment ${ }^{5}$. The purpose of the annealing process is not related to an improved crystal quality but an adjusted oxygen stoichiometry irrespective of the Ce concentration $x$. A rich interplay of magnetic and electronic phases are reported ${ }^{6-9}$ for electron doped cuprates in relation to the doping concentration $x$. In particular, the vicinity of the insulating antiferromagnetic ground state to the superconducting ground state has been investigated ${ }^{8,10-12}$ and it was found that both phases, superconducting and insulating, are in competition with each other. Moreover, the boundary between the superconducting and antiferromagnetic-insulating phases in the electronic phase diagram of electron doped cuprate superconductors is not associated to a definite value $x$, but rather varies. Li et al. ${ }^{13}$ and Charpentier et al. ${ }^{14}$ reported a critical doping concentration $x_{\mathrm{c}}=0.12$, Krockenberger et al. ${ }^{15}$ reported $x_{\mathrm{c}}=0.10$ and Brinkmann et al. reported a $x_{\mathrm{c}}=0.04{ }^{16}$ for superconducting $\mathrm{Pr}_{2-\mathrm{x}} \mathrm{Ce}_{\mathrm{x}} \mathrm{CuO}_{4}$. Furthermore, Matsumoto et al. ${ }^{17}$ reported superconductivity even for $x_{c}=0.00$ in $\mathrm{Nd}_{2-\mathrm{x}} \mathrm{Ce}_{\mathrm{x}} \mathrm{CuO}_{4}$. Although each group applies its unique annealing recipe, it is common to all five reports that the annealing conditions themselves have been kept almost constant over the entire doping range. The wide range of $x_{\mathrm{c}}$ between 0.00 and 0.12 suggests that the annealing recipe affects the competition between the antiferromagnetic insulating and superconducting states, severely. Additionally, the wide range of $x_{\mathrm{c}}$ may reflect that the appropriate annealing conditions for the induction of superconductivity themselves are doping dependent.

Electron-doped cuprate superconductors adopt the $\mathrm{T}^{\prime}$-structure $\left(\mathrm{Nd}_{2} \mathrm{CuO}_{4}\right.$ structure) where two primary sites are occupied by oxygen: $\mathrm{O}(1)$ in the $\mathrm{CuO}_{2}$ planes and $\mathrm{O}(2)$ in the rare-earth $(\mathrm{RE})$ oxide layers. Apical oxygen should not exist in the ideal $\mathrm{T}^{\prime}$ structure though they are clearly observed by Raman and far-infrared crystal-field spectroscopy ${ }^{18,19}$, Mössbauer spectroscopy ${ }^{20}$, extended X-ray absorption fine structure spectroscopy ${ }^{21,22}$ and neutron scattering ${ }^{23,24}$. An ideal annealing recipe would solely evacuate apical oxygen atoms while keeping regular oxygen sites at the $\mathrm{O}(1)$ and $\mathrm{O}(2)$ sites occupied.

\section{Results}

In this study, we used thin film $\mathrm{Pr}_{2} \mathrm{CuO}_{4}$ samples (1000 $\AA$ thick) synthesized by state-of-the-art molecular beam epitaxy (MBE). In contrast to bulk samples, the large surface-to-volume ratio of thin films along with their tenuity 
itself is advantageous in achieving homogenous oxygen configurations. Thin films of $\mathrm{Pr}_{2} \mathrm{CuO}_{4}$ have been grown intentionally at temperatures lower than optimal as a reduced crystallite dimensions are advantageous for a homogeneous annealing experience.

In general the annealing process in oxide materials is a diffusion process. In particular, regular $\mathrm{O}(1)$ and $\mathrm{O}(2)$, as well as apical $\mathrm{O}(3)$ sites are occupied or evacuated in the $\mathrm{T}^{\prime}$-cuprates. Here, we use a high precision partial oxygen pressure monitoring and control system (POPMCS) combined with X-ray diffraction and transport data of $\mathrm{Pr}_{2} \mathrm{CuO}_{4}$ for the analysis of the annealing process. A commercial quartz tube furnace equipped with a turbo molecular pump and POPMCS was used. The $\mathrm{Pr}_{2} \mathrm{CuO}_{4}$ film was mounted on the tip of a SSA-S alumina tube placed at the center of the quartz tube.

Starting from the standard annealing process (Fig. 1) typically applied to electron doped cuprates ${ }^{25,26}$, we split the annealing procedure, thus a two-step annealing process. Our systematic investigation on this new two-step annealing scheme reveals that only certain annealing conditions are suitable to preserve the $\mathrm{T}^{\prime}$-structure and induce superconductivity into $\mathrm{Pr}_{2} \mathrm{CuO}_{4}$. In Fig. 1b, we plot the electronic phase diagram of $\mathrm{Pr}_{2-\mathrm{x}} \mathrm{Ce}_{\mathrm{x}} \mathrm{CuO}_{4}$ (standard annealed), where the superconducting transition temperatures of $273 c$-axis oriented, single phase thin films of $\mathrm{Pr}_{2-\mathrm{x}} \mathrm{Ce}_{\mathrm{x}} \mathrm{CuO}_{4}$ on (001) $\mathrm{SrTiO}_{3}(\mathrm{a}=3.905$ $\AA$ ) substrates are shown for $0.00<x<0.25$. In contrast to the phase diagram for standard annealed $\mathrm{Pr}_{2-\mathrm{x}} \mathrm{Ce}_{\mathrm{x}} \mathrm{CuO}_{4}$, the ex-situ two-step annealing process allows superconductivity even without cerium. The phase diagram shown in Fig. 1c shows that superconductivity appears at all doping levels up to $x \approx 0.22$ and the highest $T_{\mathrm{c}}$ is not at $x=0.15$ but 0.00 , in stark contrast to the commonly observed phase diagram (Fig. 1b). The newly obtained superconducting phase diagram indicates that the apparent symmetry of electronic phases for hole and electron doped cuprate superconductors with respect to the antiferromagnetic-insulating ground state might be an artifact of commonly used annealing treatments, thus, not representative. Instead, it appears that for zero doping, only the T-phase is an antiferromagnetic Mott insulator whereas the $\mathrm{T}^{\prime}$-phase is a superconductor, in agreement with the first principle methods' predictions $s^{2-4}$.

\section{Discussion}

Comparing the influence of doping to the influence of annealing to $\mathrm{Pr}_{2} \mathrm{CuO}_{4}$ reveals that a hidden, hole-like Fermi surface may be present. The Ce doping dependence of the evolution of the Fermi surface of $\mathrm{Nd}_{2-\mathrm{x}} \mathrm{Ce}_{\mathrm{x}} \mathrm{CuO}_{4}$ has been reported by Armitage et al. ${ }^{27}$ for $x=0.04$, $x=0.10$ and $x=0.15$. Traces (small but finite density of states) of a hole-like Fermi surface can be detected even for $x=0.04^{27}$. However, such a sample is neither metallic nor superconducting owing to the annealing conditions applied. The hidden Fermi surface suggests that the applied annealing conditions were not optimal. Commonly, the observed Hall coefficient ${ }^{14}$ is negative for $x=0.04$. The negative Hall coefficient can be attributed to "hot spots" located at $(\pi$, $0)$ and $(0, \pi)$. The overall contribution to the Hall coefficient of those hot-spots is significant for $\operatorname{Pr}_{2-\mathrm{x}} \mathrm{Ce}_{\mathrm{x}} \mathrm{CuO}_{4}$ as the Hall coefficient is negative up to $x \approx 0.17$ (Fig. $2 \mathrm{~b}$ ). The Hall coefficients $R_{\mathrm{H}}$ taken on superconducting $\mathrm{Pr}_{2} \mathrm{CuO}_{4}$ show unambiguously that the origin of metallic conduction and superconductivity itself is not electron doping but points towards a redistribution of spectral weight from those anti-ferromagnetic "hot-spots" into the hole-like Fermi-surface ${ }^{25}$. Figure 2(c) shows the temperature dependence of $R_{\mathrm{H}}$ for superconducting $\mathrm{Pr}_{2} \mathrm{CuO}_{4}$, and the Hall coefficient of standard annealed

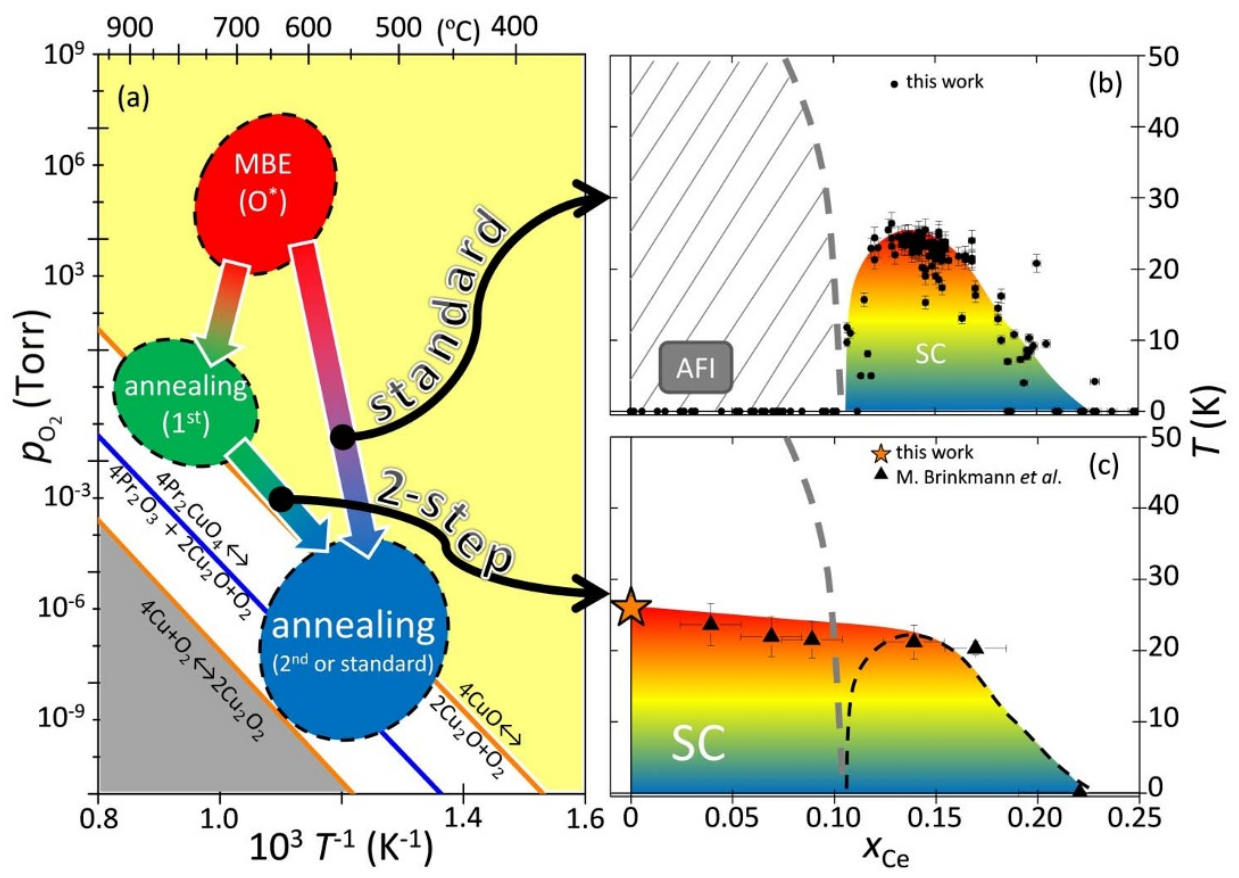

Figure $1 \mid$ Annealing paths of $\mathrm{Pr}_{2} \mathrm{CuO}_{4}$ and the resulting electronic phase diagrams. In (a), the thermodynamic phase diagram is plotted where logarithmic and reciprocal scaling is used for the oxygen pressure and the absolute temperature, respectively. Thermodynamic stability lines for the copper-oxygen system and $\mathrm{Pr}_{2} \mathrm{CuO}_{4}{ }^{52}$ are shown. $\mathrm{Pr}_{2} \mathrm{CuO}_{4}$ films were grown using a radio-frequency activated oxygen plasma $\left(\mathrm{O}^{*}\right)$ by molecular beam epitaxy. The oxygen pressure during the synthesis is $2 \times 10^{-6} \mathrm{Torr}$, corresponding to an equilibrium molecular oxygen pressure of $10^{6} \mathrm{Torr}$. The synthesis temperature of $\mathrm{Pr}_{2} \mathrm{CuO}_{4}$ is $650-750^{\circ} \mathrm{C}$. Standard annealing is carried out at temperatures between 550 and $650^{\circ} \mathrm{C}$ under $10^{-9}$ Torr. In the two-step annealing process ${ }^{53}, \mathrm{Pr}_{2-\mathrm{x}} \mathrm{Ce}_{\mathrm{x}} \mathrm{CuO}_{4}$ is annealed ex situ first at $750-850^{\circ} \mathrm{C}$ and $7.6 \times 10^{-2} \mathrm{Torr}_{2}$ and subsequently annealed at temperatures between 450 and $700^{\circ} \mathrm{C}$ under high vacuum. In (b), the doping dependence of the superconducting phase diagram of $\operatorname{Pr}_{2-\mathrm{x}} \mathrm{Ce}_{\mathrm{x}} \mathrm{CuO}_{4}$ is shown for 273 different samples obtained by the standard annealing process. For $0.00<x<0.10, \mathrm{Pr}_{2-\mathrm{x}} \mathrm{Ce}_{\mathrm{x}} \mathrm{CuO}_{4}$ is an antiferromagnetic insulator (AFI). For $0.11<x<0.23$, superconductivity is induced by the standard annealing process with a maximum $T_{\mathrm{c}}$ of $25 \mathrm{~K}$ at $x=0.14$. In (c), the doping dependence of the superconducting phase diagram of $\mathrm{Pr}_{2-\mathrm{x}} \mathrm{Ce}_{\mathrm{x}} \mathrm{CuO}_{4}$ is shown. Data points (black triangle) have been taken from ${ }^{16}$. At $x=0.00$, results of 84 samples are summarized (star). Dashed lines represent the phase diagram as obtained in Fig. 1(b). 


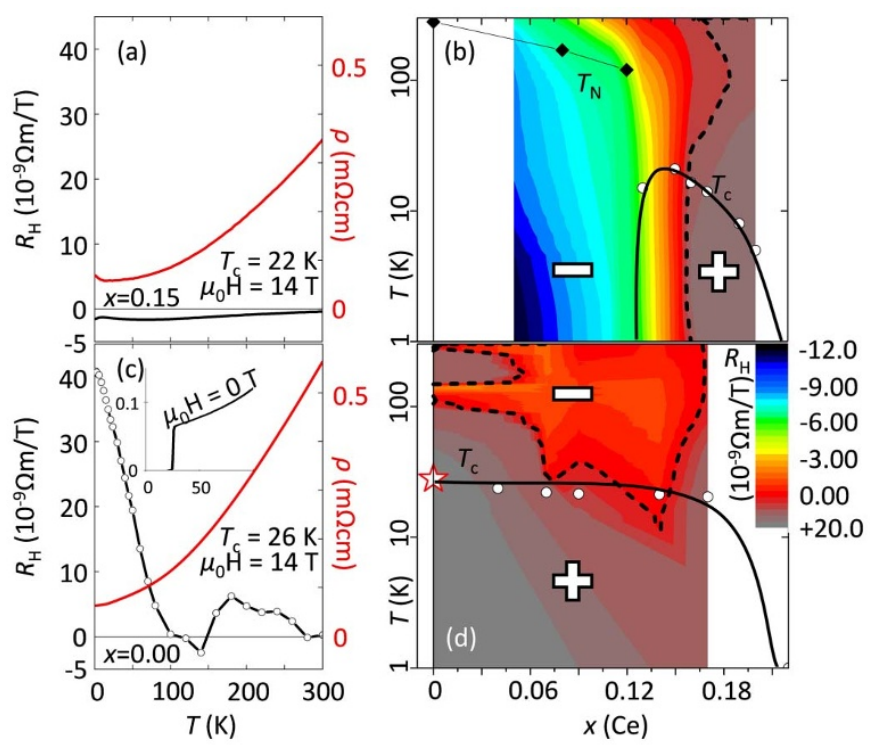

Figure $2 \mid$ For the standard annealing process (a) and (b), the highest $T_{\mathrm{c}}$ is observed for $\boldsymbol{x}=\mathbf{0 . 1 5}$. In (a) the Hall coefficient $R_{\mathrm{H}}$ (black line) and the resistivity at $\mu_{0} H=14 \mathrm{~T}$ are plotted as a function of $T$ for standard annealed $\mathrm{Pr}_{2-\mathrm{x}} \mathrm{Ce}_{\mathrm{x}} \mathrm{CuO}_{4}$ with $x=0.15$ at $\mu_{0} H=14 \mathrm{~T}$ and in (b) $R_{\mathrm{H}}$ is plotted as a function of $T$ and $x$ (data taken from ${ }^{13,54,55}$ ). Additionally, $T_{\mathrm{N}}$ and $T_{\mathrm{c}}$ are plotted as a function of $x\left(T_{\mathrm{N}}\right.$ taken from ${ }^{37}$ and $T_{\mathrm{c}}$ from $\left.{ }^{13}\right)$. The " + " and "-" marks indicate the sign of the Hall coefficient $R_{\mathrm{H}}$ and are separated by the dashed line. The cross-over from " + " to " - " is at $x \approx 0.165$ and coincides with the quantum critical point ${ }^{56}$. In case of conventionally annealed $\mathrm{Pr}_{2-\mathrm{x}} \mathrm{Ce}_{\mathrm{x}} \mathrm{CuO}_{4}$ the Hall coefficient develops monotonically upon electron doping (Ce doping) up to $x \approx 0.165$. For the 2-step annealing process (c) and (d), the highest $T_{\mathrm{c}}$ is observed at $x=0.00$ (star). In (c) $R_{\mathrm{H}}$ (black line) and the resistivity at $\mu_{0} H=14 \mathrm{~T}$ are plotted as a function of $T$ for $\mathrm{Pr}_{2} \mathrm{CuO}_{4}$ treated by a 2-step annealing process at $\mu_{0} H=14 \mathrm{~T}$ and in (d) as a function of $T$ and $x\left(T_{\mathrm{c}}\right.$ data taken from $\left.{ }^{16}\right)$. The " + " and "-" marks indicate the sign of the Hall coefficient $R_{\mathrm{H}}$ and are separated $\left(R_{\mathrm{H}}=0\right)$ by the dashed line. In case of 2-step annealed $\mathrm{Pr}_{2} \mathrm{CuO}_{4}$ the Hall coefficient is positive at $300 \mathrm{~K}$, and at $150 \mathrm{~K}$ and $120 \mathrm{~K}$, a sign change appears. Below $120 \mathrm{~K}$, the Hall coefficient is positive down to $1.7 \mathrm{~K}$. Upon electron doping (Ce doping) the low temperature Hall coefficient stays positive irrespective of the Ce concentration level. The contour-plots (b) and (d) were made from linear interpolation of $R_{\mathrm{H}}(T)$ curves for $x=0.05,0.075,0.09,0.10$, $0.12,0.14,0.15,0.17,0.19$ and $x=0.00,0.06,0.08,0.10,0.15,0.17$ in (b) and $(\mathrm{d})$, respectively.

$\operatorname{Pr}_{2-\mathrm{x}} \mathrm{Ce}_{\mathrm{x}} \mathrm{CuO}_{4}$ is shown in Figs. 2a and b. For standard annealed $\operatorname{Pr}_{2-\mathrm{x}} \mathrm{Ce}_{\mathrm{x}} \mathrm{CuO}_{4}$, the highest $T_{\mathrm{c}}$ is at $x=0.15$ and those samples have a negative $R_{\mathrm{H}}$, while $R_{\mathrm{H}}$ is positive in superconducting $\operatorname{Pr}_{2} \mathrm{CuO}_{4}$. Moreover, the Hall coefficient remains positive even after cerium doping (Fig. 2d) when an elaborate annealing process has been applied $^{16}$. In general, the Hall conductivity of a metal is expressed $^{28-30}$ as a function of the Fermi topology $d \varepsilon(\boldsymbol{k}) / d \boldsymbol{k}$ and the anisotropic relaxation time $\tau(\boldsymbol{k})$ at Fermi surface. The different signs of $R_{\mathrm{H}}$ observed for the superconducting $\mathrm{Pr}_{2} \mathrm{CuO}_{4}$ and the standard annealed superconducting $\operatorname{Pr}_{2-x} \mathrm{Ce}_{\mathrm{x}} \mathrm{CuO}_{4}$ emphasize that superconductivity in $\mathrm{Pr}_{2} \mathrm{CuO}_{4}$ is induced via another route than doping. In other words, the role of the 2-step annealing is not that of doping electron carriers via possible formation of oxygen vacancies at the regular oxygen sites. Moreover, the positive Hall coefficient of superconducting $\mathrm{Pr}_{2} \mathrm{CuO}_{4}$ reflects the situation of a hole like Fermi surface which develops upon removal of apical oxygen, which is in contrast to the hole-doped analogues, where both, the oxygenated $\mathrm{T}$ $\mathrm{La}_{2} \mathrm{CuO}_{4}+\delta$ and optimally Sr-doped $\mathrm{La}_{1.85} \mathrm{Sr}_{0.15} \mathrm{CuO}_{4}$ have positive $R_{\mathrm{H}}{ }^{31,32}$ : the additional oxygen acts as a dopant for holes and in a similar way as Sr doping. We like to highlight the fact that the temperature dependence of the Hall coefficient is not that of a simple metal but rather demonstrates the competition between a hole-like metal and an antiferromagnetic insulator. This asymmetric scenario $^{1,33-35}$ between square- and octahedral coordinated cuprates also shows that their electronic correlations are entirely different ${ }^{3}$. The absence of a doping mechanism in our elaborate annealing process is independently supported by the fact that the in-plane lattice constants of as-grown and annealed $\mathrm{Pr}_{2} \mathrm{CuO}_{4}$ films are constant upon annealing as it is well known that electron-doping stretches and holedoping shrinks the $\mathrm{Cu}-\mathrm{O}$ bonds in the $\mathrm{CuO}_{2}$ planes due to accumulation or depletion of electrons to/from the $\mathrm{Cu}-\mathrm{O} d p \sigma$ anti-bonding bands ${ }^{36}$. The presence of additional oxygen in as-grown $\mathrm{Pr}_{2} \mathrm{CuO}_{4}$ is well established ${ }^{23,24}$ as is its removal by annealing. We visualized our annealing scenario in Fig. 3a. The as-grown crystal contains more than the stoichiometric amount of oxygen which are randomly distributed at apical sites (Fig. 3a). After the first annealing step we find that the lattice parameters are nearly unchanged (Fig. 3f) when compared to the as-grown sample (Fig. 3e). However, its resistivity value is significantly higher (Fig. 3c). We explain such behavior by the introduction of oxygen vacancies in the $\mathrm{CuO}_{2}$ plane since such defects would disturb electronic conduction severely. The second annealing step does repair those in-plane defects by relocating apical oxygen atoms to the planes and consequently the resistivity is lowered significantly (Fig. 3d). This final step creates a situation similar to what has been observed after an annealing treatment ${ }^{37}$ for the cerium doped superconductors ${ }^{38}$. Overall we do observe that the $c$ axis lengths decreases upon annealing (Fig. $3 \mathrm{e}-\mathrm{g}$ ) and that has been unambiguously proven to be associated to the removal of apical oxygen by neutron scattering ${ }^{23,24}$. A typical value of the oxygen offstoichiometry estimated from neutron scattering experiments of asgrown $\mathrm{Nd}_{2} \mathrm{CuO}_{4}+\delta$ single crystals is $\delta \approx 0.05$, which indicates that one $\mathrm{Cu}$ ion out of ten unit cells is pyramidal coordinated. Experimentally, this is a sufficient condition to stabilize a long-range antiferromagnetic order even at Ce doping levels of $x=0.15^{39}$. In Ref. 39 it was shown that even for $x=0.15$ the as-grown cuprate is an antiferromagnetic insulator with a $T_{\mathrm{N}}=150 \mathrm{~K}$. After annealing, however, the cuprate system goes into the superconducting state. The only chemical difference is that occupied apical oxygen sites have been evacuated during that annealing process. Those occupied apical oxygen sites break the symmetry for all nearest and nextnearest-neighbor $\mathrm{Cu}$ plaquettes. Such a locally broken symmetry localizes electrons primarily on one $\mathrm{Cu}$ site and induce a gap in the Fermi surface. Therefore, the doping process in electron doped cuprates might be considered as a band filling process, as its ground state is already a metal ${ }^{40}$.

It is worth mentioning that the entire annealing process is a diffusion process as long as thermodynamic limits are not violated. Certainly, those limits have been violated considering earlier reports ${ }^{41}$. In contrast to the standard annealing process applied for bulk specimens, thermodynamic constraints, e.g., the $\mathrm{Pr}_{2} \mathrm{CuO}_{4} \Leftrightarrow$ $\mathrm{Pr}_{2} \mathrm{O}_{3}+\mathrm{Cu}_{2} \mathrm{O}$ stability line, may not be crossed in our 2-step annealing process. As for the standard annealing process, reduction conditions below the thermodynamic stability regions may harm the $\mathrm{T}^{\prime}$ phase, therefore $\mathrm{RE}_{2} \mathrm{O}_{3}$ oxides are often observed and consequently cause an increase of the absolute resistivity value. The annealing conditions applied in the first annealing step of our experiments are above the thermodynamic stability lines of $\mathrm{Pr}_{2} \mathrm{CuO}_{4}$ and $\mathrm{CuO}$, thus, decomposition products, i.e., $\mathrm{Pr}_{2} \mathrm{O}_{3}$, can be ruled out in contrast to other experiments as we do not see indication of their presence either by transmission electron microscopy or X-ray diffraction. Besides the influence of the annealing conditions on the electronic transport properties (Fig. 4a,b), the crystallite dimensions of the thin film are also affected. Low annealing temperatures result in larger $\left(\Delta q_{\mathrm{x}}\right)^{-1}$ values (Fig. $4 \mathrm{c}$ and $4 \mathrm{~d}$ ), though the superconducting transition temperatures are constant (Fig. 4a, 4b). Both of the annealing steps of our two-step annealing process are not independent and 


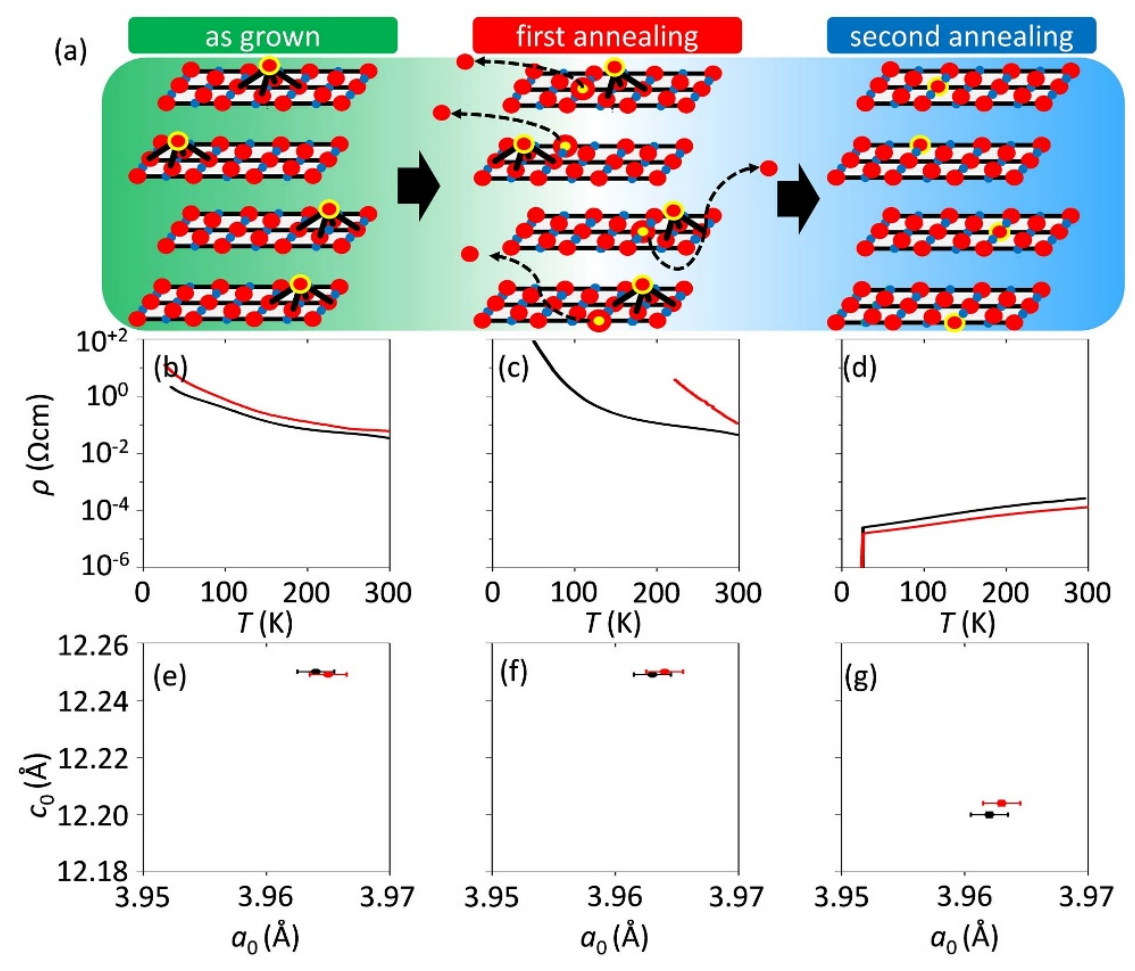

Figure 3 Schematic and simplified copper-oxygen configurations of the $\mathrm{CuO}_{2}$ planes in $\operatorname{Pr}_{2} \mathrm{CuO}_{4}$ (square planar coordinated cuprates) in accordance to the results deduced from neutron and X-ray scattering analysis and electronic transport data. (a) In the as-grown state, random apical sites of copper are occupied (apical oxygen). During the first annealing step of our two-step annealing procedure, not apical but regular oxygen sites of the $\mathrm{CuO}_{2}$ planes are being evacuated. During the second annealing step, the defective $\mathrm{CuO}_{2}$ plane is being "healed" by an oxygen rearrangement from the apical sites to regular in-plane sites (shrinkage of c-axis). (b)-(d) Evolution of $\rho$ (T) characteristics and lattice constants after each synthesis step. The asgrown $\mathrm{T}^{\prime}-\mathrm{Pr}_{2} \mathrm{CuO}_{4}$ thin film is insulating and the optimally reduced films (after step II) are superconducting while $\rho(300 \mathrm{~K})$ is reduced by more than 2 orders of magnitude. The $\mathrm{T}^{\prime}-\mathrm{Pr}_{2} \mathrm{CuO}_{4}$ thin films just after step I are even less conductive than the as-grown ones. (e)-(g) The in-plane lattice constant $\left(a_{0}\right)$ remains constant throughout the annealing process while that of $c$-axis $\left(c_{0}\right)$ shows an abrupt drop after step II. The lattice parameters $a_{0}$ and $c_{0}$ have been estimated from a Nelson-Riley function of the $(h \underline{3 h})$ and $(00 \underline{2 l})$ reflections, respectively.

their correlation to superconductivity is visualized in Fig. $4 \mathrm{e}$ where the superconducting transition temperature $\left(T_{\mathrm{c}}\right)$ is plotted as a function of the first- $\left(T_{\mathrm{a}}\right)$ and second- $\left(T_{\text {red }}\right)$ annealing temperatures. For optimal superconducting transition temperatures, a low $T_{\mathrm{a}}$ requires a low $T_{\text {red }}$ and a high $T_{\mathrm{a}}$ requires a high $T_{\text {red }}$. Consequently, when the annealing time and the oxygen partial pressures are kept constant, optimal superconducting transition temperatures are associated to $T_{\mathrm{a}}$ and $T_{\text {red }}$ in an arc shaped relation.

Finally, we compare our data to results reported from first principle calculations mentioned earlier ${ }^{2-4}$. The contrasting ground states in square-planar and octahedral coordinated cuprates, i.e., $\mathrm{T}^{\prime}$ and $\mathrm{T}$, are consequences of the difference in the charge-transfer gap $\Delta_{0}$, originating primarily from the different oxygen coordination. Vacant apical sites substantially reduce the electrostatic potential at the copper site, thus, the $3 d^{9} \mathrm{Cu}$ energy levels of the $\mathrm{T}^{\prime}$-phase are lower than in the T-phase, whereas the $2 p^{6} \mathrm{O}$ energy levels remain almost constant ${ }^{42,43}$. A simple evaluation of the unscreened $\Delta_{0}$ from Madelung potential calculations ${ }^{44,45}$ show that the difference in $\Delta_{0}$ between $\mathrm{T}^{\prime}$ - and T-phases is in the range of several $\mathrm{eV}$ - therefore, the charge transfer gap might be very small or may even vanish in the $\mathrm{T}^{\prime}$ cuprates. Under such circumstances, the model of ionic binding, which is tacitly assumed in the discussion of the charge-transfer energy, loses its vindicability. Instead, hybridization effects between $\mathrm{Cu} 3 d_{\mathrm{x}-\mathrm{y}}^{2}$ and $\mathrm{O} 2 p_{\mathrm{xy}}$ orbitals may dominate electronic correlations, though they are not taken into account in the commonly used $t$-J model ${ }^{46}$. A superconducting ground state in square planar coordinated cuprates, where doping is not a prerequisite but an option, may promote a deep understanding of the rich variety of electronic phases of cuprates as they depend on coordination, doping and diluted impurities ${ }^{47}$. Moreover, the new phase diagram of square-planar coordinated cuprates implies the following question: Does $T_{\mathrm{c}}$ further increase upon hole-doping? A recent article by Takamatsu et al. ${ }^{48}$ indeed observed superconductivity in hole doped square-planar coordinated cuprates. Answering may provide a fundamental understanding of the mechanism of high temperature superconductivity. Certainly, the induction of a long range commensurate $3 \mathrm{D}$ antiferromagnetic order by a tiny amount of apical oxygen in $\mathrm{T}^{\prime}$-cuprates demand for a thorough analysis outside of the commonly successful theoretical treatments. As the competition of antiferromagnetic and superconducting order in $\mathrm{T}^{\prime}$-cuprates ultimately tunes the electronic properties, e.g., $\rho(T), R_{\mathrm{H}}(T)$, a microscopic understanding would be beneficial. The possible solution for a quantitative analysis of site specific occupancies of oxygen in $\mathrm{T}^{\prime}$-cuprates is either via neutron scattering experiments (bulk samples) or ${ }^{17} \mathrm{O}$ nuclear magnetic resonance (NMR) spectroscopy ${ }^{49}$.

\section{Methods}

Thin films of c-axis oriented, single phase $\mathrm{Pr}_{2} \mathrm{CuO}_{4}$ were epitaxially grown on (001) $\mathrm{SrTiO}_{3}(\mathrm{a}=3.905 \AA)$ substrates by molecular beam epitaxy $(\mathrm{MBE})$. The growth of the $\mathrm{T}^{\prime}-\mathrm{Pr}_{2} \mathrm{CuO}_{4}$ films was performed in a custom-designed MBE chamber ${ }^{50,51}$ (base pressure $\sim 10^{-9}$ Torr) from metal sources by using multiple e-gun evaporators and an atomic oxygen source $(0.5 \mathrm{sccm}$, radio-frequency (RF) power of $250 \mathrm{~W})$ as an oxidizing agent. The cation stoichiometry was adjusted by controlling the evaporation beam flux of each constituent element by electron impact emission spectrometry (EIES) (Guardian IV, Inficon, USA) via feedback loops to the e-guns. Ultra-fine tuning of the evaporation beam fluxes $( \pm 0.005 \AA / \mathrm{s})$ was done by reflection highenergy electron diffraction (RHEED) monitoring ${ }^{15}$. Typically, the substrate temperature for the growth of $\mathrm{T}^{\prime}-\mathrm{Pr}_{2} \mathrm{CuO}_{4}$ thin films was $T_{\mathrm{s}}=600-650^{\circ} \mathrm{C}$. The film thickness is $1000 \AA$. For comparison purpose, some of the films were reduced in-situ after the growth under the ultra-high vacuum (UHV) environment. 

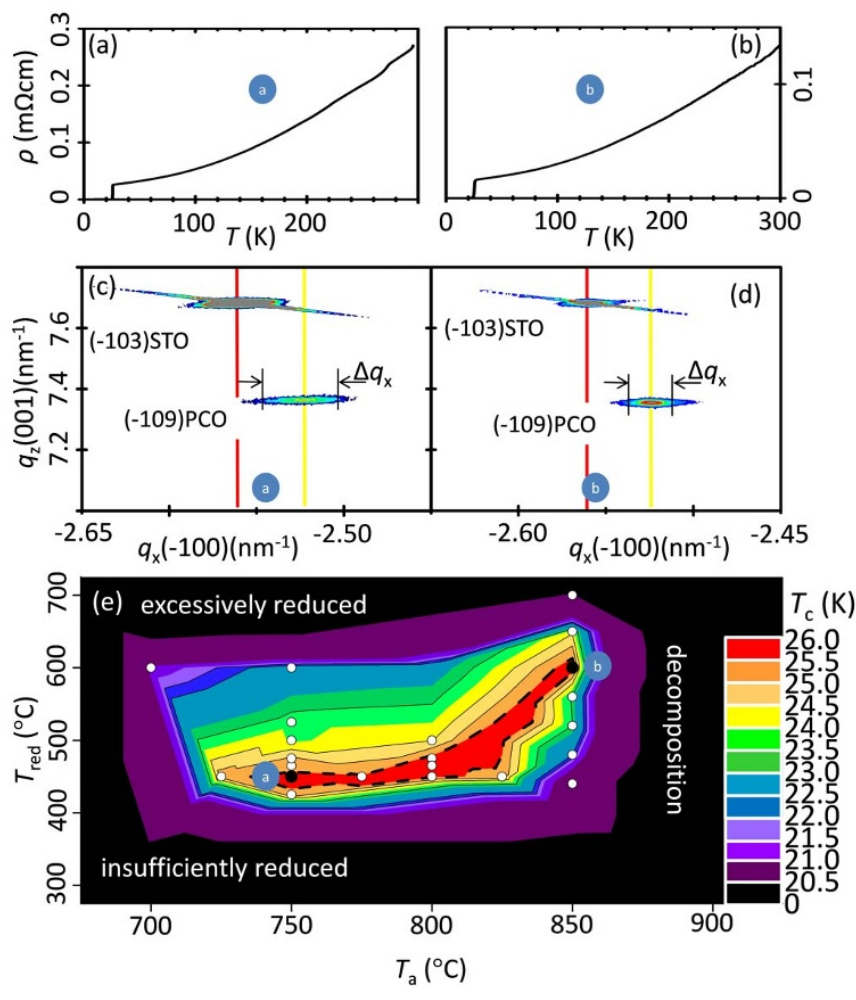

Figure $4 \mid$ The temperature dependence of the resistivity (a,b), their associated high resolution reciprocal space maps (HRRSM) of fully relaxed $\mathrm{Pr}_{2} \mathrm{CuO}_{4}$ films grown on (001) $\mathrm{SrTiO}_{3}$ substrates (c,d), and the relationship between the first $\left(T_{\mathrm{a}}\right)$ and second $\left(T_{\text {red }}\right)$ annealing temperature and their influence on the superconducting transition temperature $T_{\mathrm{c}}(\mathrm{e}) . \mathrm{In}(\mathrm{a}, \mathrm{c}), \mathrm{ar}_{2} \mathrm{CuO}_{4}$ film was annealed at $T_{\mathrm{a}}=750^{\circ} \mathrm{C}$ and $7.6 \times 10^{-2}$ Torr oxygen for $1 \mathrm{~h}$ (first annealing step), followed by a reduction process at $T_{\text {red }}=450^{\circ} \mathrm{C}$ under high vacuum for $10 \mathrm{~min}$. The electronic transport shows metallic behavior with a superconducting transition at $26.0 \mathrm{~K}$ and a residual-resistivity-ratio $(\mathrm{RRR})=7$. The relative position of the (-109) diffraction spot of $\mathrm{Pr}_{2} \mathrm{CuO}_{4}$ to the (-103) $\mathrm{SrTiO}_{3}$ diffraction spot shows that $\mathrm{Pr}_{2} \mathrm{CuO}_{4}$ films are epitaxial but relaxed grown on (001) $\mathrm{SrTiO}_{3}$. The in-plane lattice constant of the $\mathrm{Pr}_{2} \mathrm{CuO}_{4}$ films is 3.96 A. $\left(\Delta q_{\mathrm{x}}\right)^{-1} \approx 80 \mathrm{~nm}$ provides a rough estimation of the lateral crystallite dimensions. In (b, d), a $\mathrm{Pr}_{2} \mathrm{CuO}_{4}$ film was annealed at $T_{\mathrm{a}}=850^{\circ} \mathrm{C}$ and 7.6 $\times 10^{-2}$ Torr oxygen for $1 \mathrm{~h}$ (first annealing step), followed by a reduction process at $T_{\text {red }}=650^{\circ} \mathrm{C}$ under high vacuum for $10 \mathrm{~min}$. The electronic transport shows metallic behavior with a superconducting transition at 25.0 Kand RRR $>5$. The relative position of the $(-109)$ diffraction spot of $\mathrm{Pr}_{2} \mathrm{CuO}_{4}$ to the $(-103) \mathrm{SrTiO}_{3}$ diffraction spot shows that $\mathrm{Pr}_{2} \mathrm{CuO}_{4}$ films are epitaxial but relaxed grown on (001) $\mathrm{SrTiO}_{3}$. The in-plane lattice constant of the $\mathrm{Pr}_{2} \mathrm{CuO}_{4}$ films is $3.96 \AA .\left(\Delta q_{\mathrm{x}}\right)^{-1} \approx 250 \mathrm{~nm}$ provides a rough estimation of the lateral crystallite dimensions. The influence of the annealing history on the superconducting transition temperature $T_{\mathrm{c}}$ is given in (e). Here, the oxygen partial pressures during the first and second annealing steps were kept constant and are $7.6 \times 10^{-2}$ Torr and high vacuum, respectively. $T_{\mathrm{c}}$ levels as high as $26.0 \mathrm{~K}$ can be reached for $\mathrm{Pr}_{2} \mathrm{CuO}_{4}$ films grown on (001) $\mathrm{SrTiO}_{3}$ substrates.

Using the MBE-grown films, we investigated the reduction condition dependence of the properties of $\mathrm{T}^{\prime}-\mathrm{Pr}_{2} \mathrm{CuO}_{4}$. A commercial quartz tube furnace of $60 \mathrm{~cm}$ length and $30 \mathrm{~mm}$ diameter was used. The furnace is equipped with a turbo molecular pump (TMP) and a commercial (SiOC-200, STLAB, Japan) high precision partial oxygen pressure monitoring and control system (POPMCS). The POPMCS allows a precise control of the oxygen partial pressure between $10^{-1}$ to $10^{-16} \mathrm{~atm}$ by mixing an inert gas, e.g., $\mathrm{N}_{2}$, and oxygen at an electrochemically controlled oxygen diffusor (yttrium stabilized zirconium oxide). The $\mathrm{Pr}_{2} \mathrm{CuO}_{4}$ film was mounted on the tip of a SSA-S alumina tube placed at the center of the quartz tube in longitudinal direction. Prior to its first usage the quartz tube was cleaned in boiling piranha clean whereas the alumina tube was rinsed by deionized water. The cleaned quartz tube and SSA-S alumina tube were prebaked at $1000^{\circ} \mathrm{C}$ for $10 \mathrm{~h}$ under ultra-high vacuum. Prior to the first annealing step, the partial pressure of oxygen was adjusted to a defined value. The $\mathrm{N}_{2} / \mathrm{O}_{2}$ gas mixture was kept at a constant flow rate of $500 \mathrm{sccm}$ throughout all experiments. The second annealing step is performed in the same tubular furnace evacuated in $10^{-5}$ Torr residual gas pressure.

1. Dagotto, E. Correlated electrons in high-temperature superconductors. Reviews of Modern Physics 66, 763-840 (1994).

2. Weber, C., Haule, K. \& Kotliar, G. Apical oxygens and correlation strength in electron- and hole-doped copper oxides. Physical Review B 82, 125107 (2010).

3. Weber, C., Haule, K. \& Kotliar, G. Strength of correlations in electron- and holedoped cuprates. Nature Physics 6, 574-578 (2010).

4. Das, H. \& Saha-Dasgupta, T. Electronic structure of $\mathrm{La}_{2} \mathrm{CuO}_{4}$ in the $\mathrm{T}$ and $\mathrm{T}$ ' crystal structures using dynamical mean field theory. Physical Review B 79, 134522 (2009).

5. Tokura, Y., Takagi, H. \& Uchida, S. A superconducting copper oxide compound with electrons as the charge carriers. Nature 337, 345 (1989).

6. Kang, H. J. et al. Antiferromagnetic order as the competing ground state in electron-doped $\mathrm{Nd}_{1.85} \mathrm{Ce}_{0.15} \mathrm{CuO}_{4}$. Nature 423, 522-525 (2003).

7. Motoyama, E. M. et al. Spin correlations in the electron-doped high-transitiontemperature superconductor $\mathrm{Nd}_{2-\mathrm{x}} \mathrm{Ce}_{\mathrm{x}} \mathrm{CuO}_{4}$. Nature 445, 186-189 (2007).

8. Wilson, S. D. et al. Resonance in the electron-doped high-transition-temperature superconductor $\mathrm{Pr}_{0.88} \mathrm{LaCe}_{0.12} \mathrm{CuO}_{4}$. Nature 442, 59-62 (2006).

9. Jin, K., Butch, N. P., Kirshenbaum, K., Paglione, J. \& Greene, R. L. Link between spin fluctuations and electron pairing in copper oxide superconductors. Nature 476, 73-75 (2011).

10. Mang, P. K., Vajk, O. P., Arvanitaki, A., Lynn, J. W. \& Greven, M. Spin correlations and magnetic order in non superconducting $\mathrm{Nd}_{2-\mathrm{x}} \mathrm{Ce}_{\mathrm{x}} \mathrm{CuO}_{4} \pm \delta$. Physical Review Letters 93, 027002 (2004).

11. Lee, P. A., Nagaosa, N. \& Wen, X.-G. Doping a Mott insulator: Physics of hightemperature superconductivity. Reviews of Modern Physics 78, 17-85 (2006).

12. Damascelli, A., Hussain, Z. \& Shen, Z.-X. Angle-resolved photoemission studies of the cuprate superconductors. Reviews of Modern Physics 75, 473-541 (2003).

13. Li, P., Balakirev, F. F. \& Greene, R. L. High-Field Hall Resistivity and Magnetoresistance of Electron-Doped $\mathrm{Pr}_{2-\mathrm{x}} \mathrm{Ce}_{\mathrm{x}} \mathrm{CuO}_{4-\delta}$. Physical Review Letters 99, 047003 (2007).

14. Charpentier, S. et al. Antiferromagnetic fluctuations and the Hall effect of electron-doped cuprates: Possibility of a quantum phase transition at underdoping. Physical Review B 81, 104509 (2010).

15. Krockenberger, Y. et al. Superconductivity phase diagrams for the electron-doped cuprates $R_{2-\mathrm{x}} \mathrm{Ce}_{\mathrm{x}} \mathrm{CuO}_{4}(R=\mathrm{La}, \mathrm{Pr}, \mathrm{Nd}, \mathrm{Sm}$, and Eu). Physical Review B 77, 060505 (2008).

16. Brinkmann, M., Rex, T., Bach, H. \& Westerholt, K. Extended Superconducting Concentration Range Observed in $\operatorname{Pr}_{2-\mathrm{x}} \mathrm{Ce}_{\mathrm{x}} \mathrm{CuO}_{4-\delta}$. Physical Review Letters 74, 4927-4930 (1995).

17. Matsumoto, O. et al. Generic phase diagram of "electron-doped" T' cuprates. Physica C: Superconductivity 469, 924-927 (2009).

18. Riou, G. et al. $\mathrm{Pr}^{3+}$ crystal-field excitation study of apical oxygen and reduction processes in $\mathrm{Pr}_{2-\mathrm{x}} \mathrm{Ce}_{\mathrm{x}} \mathrm{CuO}_{4} \pm \delta$. Physical Review B 69, 024511 (2004).

19. Richard, P. et al. Role of oxygen nonstoichiometry and the reduction process on the local structure of $\mathrm{Nd}_{2-\mathrm{x}} \mathrm{Ce}_{\mathrm{x}} \mathrm{CuO}_{4} \pm \delta$. Physical Review B 70, 064513 (2004).

20. Nath, A. et al. Chemical Signature of the Superconducting Phase in the Nd-CeCu-O System. Science 265, 73-74 (1994).

21. Ghigna, P. et al. Local structure around Ce in the $\mathrm{Nd}_{2-\mathrm{x}} \mathrm{Ce}_{\mathrm{x}} \mathrm{CuO}_{4}$ superconductor probed by EXAFS. Eur. Phys. J. B 41, 31-42 (2004).

22. Tanaka, Y. et al. Quantitative XANES Spectroscopy Study on the Prototype Holeand Electron-Doped High-Tc Superconductor Systems, $(\mathrm{La}, \mathrm{Sr})_{2} \mathrm{CuO}_{4}$ and $(\mathrm{Nd}, \mathrm{Ce})_{2} \mathrm{CuO}_{4}$. Chemistry of Materials 20, 5414-5420 (2008).

23. Schultz, A. J., Jorgensen, J. D., Peng, J. L. \& Greene, R. L. Single-crystal neutrondiffraction structures of reduced and oxygenated $\mathrm{Nd}_{2-\mathrm{x}} \mathrm{Ce}_{\mathrm{x}} \mathrm{CuO}_{\mathrm{y}}$. Physical Review B 53, 5157-5159 (1996).

24. Radaelli, P. G., Jorgensen, J. D., Schultz, A. J., Peng, J. L. \& Greene, R. L. Evidence of apical oxygen in $\mathrm{Nd}_{2} \mathrm{CuO}_{\mathrm{y}}$ determined by single-crystal neutron diffraction. Physical Review B 49, 15322-15326 (1994).

25. Armitage, N. P., Fournier, P. \& Greene, R. L. Progress and perspectives on electron-doped cuprates. Reviews of Modern Physics 82, 2421-2487 (2010).

26. Krockenberger, Y., Yamamoto, H., Tsukada, A., Mitsuhashi, M. \& Naito, M. Unconventional transport and superconducting properties in electron-doped cuprates. Physical Review B 85, 184502 (2012).

27. Armitage, N. P. et al. Doping Dependence of an n-Type Cuprate Superconductor Investigated by Angle-Resolved Photoemission Spectroscopy. Physical Review Letters 88, 257001 (2002).

28. Hurd, C. M. The Hall effect in metals and alloys (Plenum Press, 1972).

29. Ong, N. P. Geometric interpretation of the weak-field Hall conductivity in twodimensional metals with arbitrary Fermi surface. Physical Review B 43, 193-201 (1991).

30. Nair, S. et al. Hall effect in heavy fermion metals. Advances in Physics 61, 583-664 (2012).

31. Hirayama, T., Nakagawa, M. \& Oda, Y. Hall effect in electrochemically oxidized $\mathrm{La}_{2} \mathrm{CuO}_{4}+\delta$ and $\left(\mathrm{La}_{1-\mathrm{x}} \mathrm{Sr}_{\mathrm{x}}\right)_{2} \mathrm{CuO}_{4+\delta}$. Physica C: Superconductivity 334, 289-294 (2000). 
32. Poccia, N. et al. Optimum inhomogeneity of local lattice distortions in $\mathrm{La}_{2} \mathrm{CuO}_{4}+\mathrm{y}$. Proceedings of the National Academy of Sciences 109, 15685-15690 (2012)

33. Imada, M., Fujimori, A. \& Tokura, Y. Metal-insulator transitions. Reviews of Modern Physics 70, 1039-1263 (1998).

34. Pickett, W. E. Electronic structure of the high-temperature oxide superconductors. Reviews of Modern Physics 61, 433-512 (1989).

35. Kastner, M. A., Birgeneau, R. J., Shirane, G. \& Endoh, Y. Magnetic, transport, and optical properties of monolayer copper oxides. Reviews of Modern Physics 70, 897-928 (1998).

36. Wang, E., Tarascon, J. M., Greene, L. H., Hull, G. W. \& McKinnon, W. R. Cationic substitution and role of oxygen in the $n$-type superconducting $\mathrm{T}^{\prime}$ system $\mathrm{Nd}_{2-\mathrm{y}} \mathrm{Ce}_{\mathrm{y}} \mathrm{CuO}_{\mathrm{z}}$. Physical Review B 41, 6582-6590 (1990).

37. Thurston, T. R. et al. Antiferromagnetic spin correlations in $(\mathrm{Nd}, \mathrm{Pr})_{2-\mathrm{x}} \mathrm{Ce}_{\mathrm{x}} \mathrm{CuO}_{4-\delta}$ Physical Review Letters 65, 263-266 (1990).

38. King, D. M. et al. Fermi surface and electronic structure of $\mathrm{Nd}_{2-\mathrm{x}} \mathrm{Ce}_{\mathrm{x}} \mathrm{CuO}_{4-\delta}$. Physical Review Letters 70, 3159-3162 (1993).

39. Matsuda, M. et al. Magnetic order, spin correlations, and superconductivity in single-crystal $\mathrm{Nd}_{1.85} \mathrm{Ce}_{0.15} \mathrm{CuO}_{4}+\delta$. Physical Review B 45, 12548-12555 (1992).

40. Sebastian, S. E. et al. Metal-insulator quantum critical point beneath the high $T_{\mathrm{c}}$ superconducting dome. Proceedings of the National Academy of Sciences 107, 6175-6179 (2010).

41. Kang, H. J. et al. Microscopic annealing process and its impact on superconductivity in T'-structure electron-doped copper oxides. Nat Mater 6, 224-229 (2007).

42. Koitzsch, A. et al. Core-hole screening response in two-dimensional cuprates: A high-resolution X-ray photoemission study. Physical Review B 66, 024519 (2002).

43. Patabendige, C. N. K., Azad, A. K., Connor, P. A., Rolle, A. \& Irvine, J. T. S. Remarkable transition from rocksalt/perovskite layered structure to fluorite/ rocksalt layered structure in rapidly cooled $\mathrm{Ln}_{2} \mathrm{CuO}_{4}$. Sci. Rep. 3 (2013).

44. Zhou, J. S., Chan, J. \& Goodenough, J. B. Copper-oxygen bond length and selfdoping in $R_{2} \mathrm{CuO}_{4}(R=\mathrm{Pr}, \mathrm{Nd}, \mathrm{Sm}, \mathrm{Eu}, \mathrm{Gd})$. Physical Review B 47, 5477-5480 (1993).

45. Tsukada, A., Shibata, H., Noda, M., Yamamoto, H. \& Naito, M. Charge transfer gap for $\mathrm{T}^{\prime}-\mathrm{RE}_{2} \mathrm{CuO}_{4}$ and $\mathrm{T}-\mathrm{La}_{2} \mathrm{CuO}_{4}$ as estimated from Madelung potential calculations. Physica C: Superconductivity 445-448, 94-96 (2006).

46. Feiner, L. F., Jefferson, J. H. \& Raimondi, R. Effective single-band models for the high- $T_{\mathrm{c}}$ cuprates. I. Coulomb interactions. Physical Review B 53, 8751-8773 (1996)

47. Wang, Y. \& Ong, N. P. Particle-hole symmetry in the antiferromagnetic state of the cuprates. Proceedings of the National Academy of Sciences 98, 11091-11096 (2001).

48. Tomohisa Takamatsu, Masatsune Kato, Takashi Noji \& Koike, Y. Undoped and Hole-Doped Superconductors ' $\mathrm{T}^{\prime}-\mathrm{La}_{1.8-\mathrm{x}} \mathrm{Eu}_{0.2} \mathrm{Sr}_{\mathrm{x}} \mathrm{CuO}_{4}(\mathrm{x}=0$ and 0.05) Prepared by Solid-State Reaction. Applied Physics Express 5, 073101 (2012).

49. Rigamonti, A., Borsa, F. \& Carretta, P. Basic aspects and main results of NMRNQR spectroscopies in high-temperature superconductors. Reports on Progress in Physics 61, 1367 (1998).

50. Naito, M. \& Sato, H. Stoichiometry control of atomic beam fluxes by precipitated impurity phase detection in growth of $(\mathrm{Pr}, \mathrm{Ce})_{2} \mathrm{CuO}_{4}$ and $(\mathrm{La}, \mathrm{Sr})_{2} \mathrm{CuO}_{4}$ films. Applied Physics Letters 67, 2557-2559 (1995)
51. Naito, M., Sato, H. \& Yamamoto, H. MBE growth of $(\mathrm{La}, \mathrm{Sr})_{2} \mathrm{CuO}_{4}$ and (Nd,Ce) ${ }_{2} \mathrm{CuO}_{4}$ thin films. Physica C: Superconductivity 293, 36-43 (1997).

52. Petrov, A. N., Zuev, A. Y. \& Cherepanov, V. A. Thermodynamic stability of the lanthanide cuprates $\mathrm{Ln}_{2} \mathrm{CuO}_{4}$ and $L n \mathrm{CuO}_{2}$, where $\mathrm{Ln}=\mathrm{La}, \mathrm{Pr}, \mathrm{Nd}, \mathrm{Sm}$, Eu, or Gd Russ. J. Phys. Chem. 62, 1613-1615 (1988).

53. Yamamoto, H., Matsumoto, O., Krockenberger, Y., Yamagami, K. \& Naito, M. Molecular beam epitaxy of superconducting $\mathrm{Pr}_{2} \mathrm{CuO}_{4}$ films. Solid State Communications 151, 771-774 (2011).

54. Dagan, Y., Qazilbash, M. M., Hill, C. P., Kulkarni, V. N. \& Greene, R. L. Evidence for a Quantum Phase Transition in $\mathrm{Pr}_{2-\mathrm{x}} \mathrm{Ce}_{\mathrm{x}} \mathrm{CuO}_{4-\delta}$ from Transport Measurements. Physical Review Letters 92, 167001 (2004).

55. Gauthier, J. et al. Different roles of cerium substitution and oxygen reduction in transport in $\mathrm{Pr}_{2-\mathrm{x}} \mathrm{Ce}_{\mathrm{x}} \mathrm{CuO}_{4}$ thin films. Physical Review B 75, 024424 (2007).

56. Butch, N. P., Jin, K., Kirshenbaum, K., Greene, R. L. \& Paglione, J. Quantum critical scaling at the edge of Fermi liquid stability in a cuprate superconductor. Proceedings of the National Academy of Sciences 109, 8440-8444 (2012).

\section{Acknowledgements}

The authors are grateful to Dirk Manske, Paul Grant, George Sawatzki, Andrea Damascelli, and James Analytis. H. Y. thanks Hiroyuki Shibata, Yoshitaka Taniyasu and Tetsuya Akasaka for their support in experiments. H. Y. also acknowledges fruitful discussions on oxygen engineering in oxides with Masaru Tsuchiya. The work performed at Tokyo University of Agriculture and Technology was partially supported by KAKENHI B (Grant No. 18340098) from Japan Society for the Promotion of Science (JSPS).

\section{Author contributions}

All the MBE samples for ex-situ annealing experiments were prepared by H.Y. or Y.K. O.M., K.Y. and M.M. performed the ex-situ post-annealing experiments as well as most of the $\mathrm{XRD}, \mathrm{AFM}$, and $\rho(T)$ measurements. In-situ annealing experiments were done by A.T. Y.K. carried out XRD experiments as well as characterization of magnetic properties by using a SQUID magnetometer. H.I. prepared the Hall bars and H.I. and Y.K. performed measurements of the Hall coefficient. M.N. along with other authors discussed the results and commented on the paper. All work was coordinated and overseen by H.Y. and M.N.

\section{Additional information}

Supplementary information accompanies this paper at http://www.nature.com/ scientificreports

Competing financial interests: The authors declare no competing financial interests How to cite this article: Krockenberger, Y. et al. Emerging superconductivity hidden beneath charge-transfer insulators. Sci. Rep. 3, 2235; DOI:10.1038/srep02235 (2013). To view a copy of this license, visit http://creativecommons.org/licenses/by/3.0 